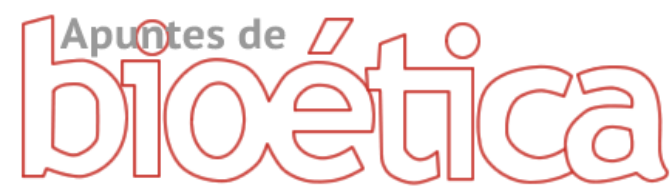

https://doi.org/10.35383/apuntes.v3i1.366

\title{
Un enfoque personalista ontológico de los dilemas surgidos en la pandemia COVID-19
}

\author{
Moya G. ${ }^{1}$
}

\section{INFORMACIÓN DEL ARTÍCULO RESUMEN}

Historia del artículo:

Recibido el 22 de abril 2020

Aceptado el 23 de julio 2020

\section{Palabras claves:}

Personalismo ontológico,

Pandemia COVID-19,

Dignidad,

Principios Éticos.

La pandemia COVID-19 ha dejado al descubierto, en forma dramática, la falta de inversión y gestión en el sistema de salud y las debilidades estructurales del sistema de protección social. El análisis de los dilemas que surgen como secuencia de la toma de decisiones para lidiar contra los efectos de la pandemia puede dejar de lado la protección y promoción del bienestar de la persona como centro de la organización de la sociedad, y centrarse solo en el bienestar colectivo e institucional. El personalismo ontológico como teoría bioética permite un análisis profundo de estos dilemas, ya que su base filosófica se centraliza en la defensa y promoción de la persona, y en la búsqueda del bien común. En este artículo se analizarán los fundamentos de esta corriente bioética y las respuestas que puede aportar a los dilemas morales expuestos en forma intempestiva por la pandemia COVID-19.

\section{Título en inglés}

\section{ABSTRACT}

\section{Keywords.}

Ontologic Personalism

COVID-19 pandemia

Dignity

Ethical Principles
The COVID-19 pandemic has dramatically exposed the lack of investment and management in the health system and the structural weaknesses of the social protection. The analysis of the dilemmas that arise as a sequence of decision-making to deal with the effects of the pandemic can leave aside the protection and promotion of the well-being of the person as the center of the organization of society, and focus only on the collective and institutional well-being. Ontological personalism as a bioethical theory allows a deep analysis of these dilemmas, since its philosophical base is centralized in the defense and promotion of the person, and in the search for the common good. This article analyzes the foundations of this bioethical current and the responses it can provide to the moral dilemmas exposed untimely by the COVID-19 pandemic.

1 Doctora en Biomedicina, Magister en Bioética, Magister en Biología Molecular Médica, Médica Genetista. Instituto de Bioética- Facultad de Ciencias Médicas Universidad Católica Argentina, Buenos Aires, Argentina Miembro correspondiente de la Pontificia Academia Por-Vita Email: gracielamoya@uca.edu.ar ORCID: https://orcid.org/0000-0001-9412-3471 


\section{Introducción}

Las pandemias son enfermedades epidémicas que se propagan abruptamente en forma mundial o afectan a casi todos los individuos de una localidad o región. Esta rápida propagación ocurre porque es una infección nueva frente a la cual las personas no tienen inmunidad propia o adquirida mediante una vacuna, y generan síntomas graves que pueden asociarse a una mayor tasa de mortalidad en personas de grupos de riesgo más susceptibles por condiciones de salud previas, en determinados momentos de su ciclo vital, o en situaciones de mayor vulnerabilidad social. Anteriormente hemos visto que existe una mayor susceptibilidad en ciertos grupos poblacionales en diferentes epidemias. Entre ellas, la epidemia de poliomielitis que comenzó a fines del siglo XIX en muchos países europeos y América del Norte, luego de lo cual se convirtió en una enfermedad global con epidemias anuales, afectando principalmente a niños pequeños (Nathanson \& Kew, 2010). La introducción de los distintos tipos de vacunas contra esta infección ha evitado la ocurrencia de epidemias masivas, y actualmente existen distintos programas de vacunación que tienen la finalidad de erradicar en forma definitiva esta infección (Pagliusi, Dennehy, Kim, \& DCVMN AGM Organizing Committee).

Las mujeres embarazadas y sus niños en gestación representan una población de alto riesgo en otros brotes de infecciones virales. Durante la gestación existen cambios en el sistema inmune de la madre para proteger al feto, ${ }^{2}$ que dejan a la mujer en situación de mayor vulnerabilidad para infecciones virales ( Dashraath, y otros, 2020) (Schwartz \& Graham, 2020, pág. 194). En el año 2005 se detectó en Brasil un brote provocado por el virus del Zika (ZIKA, arbovirus de la familia Flavivirus). La

${ }^{2}$ El sesgo del embarazo hacia el dominio del sistema T-helper 2 (Th2) que protege al feto, deja a la madre vulnerable a las infecciones virales, que están más efectivamente contenidas por el sistema Th1. infección se asoció con una alta incidencia de microcefalia y otras anormalidades del sistema nervioso central en los recién nacidos, expuestos a esta infección durante la gestación (Hajra, Bandyopadhyay, \& Hajra, 2016), hubo más de 4.000 casos de microcefalia y trastornos neurológicos en algunas áreas afectadas por el virus Zika (Hajra, Bandyopadhyay, \& Hajra, 2016). Si bien, alrededor del $80 \%$ de los casos los pacientes son asintomáticos, el niño en gestación es altamente susceptible a los efectos de esta infección que se ha asociado con un riesgo elevado de microcefalia, malformaciones cerebrales y retardo del crecimiento intrauterino (Boeuf, Drummer, Richards, Scoullar, \& Beeson, 2016, pág. 112). Por el momento, no hay ningún tratamiento efectivo durante la infección o vacuna que la prevenga, por ello sólo están las medidas preventivas que eviten la infección transmitida por el mosquito Aedes spp, o por vía sexual (Hajra, y otros, 2017). Dado el masivo impacto que provoca esta infección en zonas de riesgo, y especialmente las consecuencias a largo plazo, en febrero de 2016 la Organización Mundial de la Salud (OMS) la declara como una emergencia de salud pública de preocupación internacional, colocándola en la misma lista de prioridades que el brote del virus del Ébola (Boeuf, Drummer, Richards, Scoullar, \& Beeson, 2016).

La pandemia de la gripe H1N1 (SARS) producida por el virus de la influenza porcina $A$ en 2009-2010 que ha afectado más severamente a las mujeres embarazadas, en el segundo y tercer trimestre, o a aquellas que tenían condiciones previas de salud (ACOG, 2003). Durante la gestación ocurren cambios fisiológicos, no sólo se encuentran en un estado de inmunosupresión para proteger al feto como se refirió previamente, sino que también, tienen cambios respiratorios adaptativos que las vuelven más sensibles a la hipoxia (Pringle, Kind, Sferruzzi-Perri, Thompson, \& Roberts, 2010), por lo que son más susceptibles a patógenos respiratorios y en consecuencia a formas más 
severas de neumonía. En la infección por el virus de la gripe $\mathrm{A}$ (H1N1) se reportó que las mujeres embarazadas tenían cuatro veces más probabilidades de ser ingresadas en el hospital que la población general (Bauch, Lloyd-Smith, Coffee, \& Galvani, 2005), y aproximadamente el $50 \%$ de las mujeres embarazadas con SARS requirieron cuidados intensivos, y alrededor del $33 \%$ de ellas necesitó ventilación mecánica, con una tasa de mortalidad cercana al 25\% (Cascella, Rajnik, Cuomo, Dulebohn, \& Di Napoli, 2020). Una de las causas que determinó estas tasas fue el retraso en el inicio del tratamiento por negativa de la paciente o duda del médico (Rasmussen, y otros, 2009), debido a la falta de información sobre los efectos en la mujer embarazada o en el niño por nacer.

En marzo de 2020 la Organización Mundial de la Salud declaró pandemia a la enfermedad conocida como COVID-19, causada por el coronavirus SARS-CoV-2 debido a que, por su alta contagiosidad ha provocado una rápida escalada en el número de personas infectadas a nivel mundial (Organización Mundial de la Salud, 2020). Hasta el 10 de junio, se habían notificado más de 7.145.539 casos y 408.025 muertes en todo el mundo (World Health Organization, 2020).

Las manifestaciones clínicas de COVID-19 son muy variables, ya que puede presentarse en forma asintomática o sintomática: incluyendo cuadros respiratorios que varían desde tos, malestar general y fiebre hasta neumonía grave con síndrome de distrés respiratorio agudo, shock séptico y fallo multi-orgánico (Huang, y otros, 2020) (China Medical Treatment Expert Group for Covid-19, 2020).

Los reportes revelan que existe una población de mayor riesgo de sufrir complicaciones severas por COVID-19, entre ellos pacientes con enfermedades cardiovasculares, enfermedad pulmonar obstructiva crónica, diabetes, demencia, cáncer activo en los últimos cinco años, enfermedad hepática o renal crónica, especialmente si algunas de estas enfermedades coexisten en el mismo paciente (Istituto Superiore di Sanit, 2020).

Debido a la alta contagiosidad, la mayor mortalidad en aquellos grupos de riesgo, la mayor posibilidad de contagio del personal sanitario, de seguridad, de transporte o asistencia social, y el colapso provocado en el sistema de salud, se han tomado en la mayoría de los países medidas médicas y no médicas para lidiar con la pandemia que pueden poner en riesgo la defensa de la vida de la persona humana, o generar situaciones de desprotección en los grupos en situación de vulnerabilidad (Markel, H, y otros, 2007). Es claro que una pandemia comienza como un problema de salud de las personas, se convierte en un problema que involucra a la salud pública, y finalmente su impacto es más profundo, afectando todas las instancias de la vida en sociedad. Las manifestaciones de salud que genera la pandemia y las medidas que son necesarias tomar para su control se transforman en dilemas que perturban inicialmente lo individual para luego tener efecto comunitario en todas las áreas sociales (Jones, 2020).

Es importante encontrar un equilibrio entre las acciones que se tomen para lidiar contra la pandemia y la defensa y promoción de la persona humana, y su vida en sociedad.

\section{Resultados, análisis y discusión}

\section{Acciones para lidiar contra la pandemia y sus consecuencias}

En pocas semanas el brote iniciado como una nueva enfermedad respiratoria severa en Wuhan, Provincia de Hubei, China, en diciembre de 2019, se convirtió en una pandemia global debido a cinco características de esta enfermedad: la alta contagiosidad de persona a persona; una alto porcentaje de personas asintomáticas, portadoras del virus, que lo 
diseminan; la severidad de los síntomas, que requieren cuidados de salud de alta complejidad (20\% de los casos son pacientes en estado crítico), y con una alta mortalidad (alrededor del $3 \%$ en aquellas personas de alto riesgo de complicaciones; la falta de inmunidad natural que nos convierte a todos susceptibles de infección; y finalmente, aún no se cuenta con un tratamiento efectivo o vacuna que prevenga el contagio. Esto ha llevado a que los sistemas de salud, aún en países desarrollados se hallan visto superados y colapsados respecto de sus recursos humanos, de infraestructura y económicos; y los profesionales de la salud ejerzan su atención en una angustiante interfase entre la asistencia y la investigación, sin respuestas seguras sobre el tratamiento, la curación o las secuelas, con información escasa y contradictoria.

En un esfuerzo conjunto la OMS, los gobiernos y sus autoridades de salud pública están intentando, con mayor o menor éxito, establecer por un lado líneas de investigación que definan el tratamiento más efectivo para los pacientes en las distintas etapas de la enfermedad, y por otro, tomar medidas que aplanen la curva de contagios de COVID-19 con la finalidad de evitar el colapso de los sistemas de salud (World Health Organization , 2020). Muchos países han considerado esta pandemia como una amenaza seria e inminente para la salud pública, lo que ha habilita al estado a tomar medidas de control sobre la población, como es la restricción en el movimiento de las personas, tanto para las personas infectadas como para aquellas que aún no se han infectado.

Se reconocen que ciertas indicaciones son más efectivas para limitar la propagación de una pandemia y para mantener el riesgo de la salud pública lo más bajo posible (Homeland Security Council, 2005). Estas medidas, implican un esfuerzo conjunto ya que incorpora intervenciones de la ciudadanía, como es la adhesión a las medidas de control de infecciones (higiene de manos y superficie, uso de guantes y barbijos para reducir la propagación del virus), distanciamiento social, aislamiento de las personas con más riesgo de formas graves, reducción del transporte público y cierre fronterizo. Si bien muchas de esas intervenciones tratan de crear condiciones que permitan reducir la tasa de infectados y por ende disminuir la mortalidad, tienen un impacto en la privacidad, libertad o albedrío de las personas, y un alto costo en el sistema productivo y económico de un país. Estas medidas pueden exacerbar los conflictos sociales ya existentes al incrementar el control del estado sobre los ciudadanos, interpretándose como medidas de coerción e intrusión en la vida de las personas.

\section{Capacidad del sistema de salud:}

El sistema de salud tiene recursos limitados, tanto a nivel de los profesionales, como de los centros de internación, equipamiento de las terapias intensivas, equipos de protección personal, y todas las necesidades que cubran las intervenciones diagnósticas, terapéuticas y preventivas. Cada país organiza la posibilidad de realizar el testeo, la internación, o el cuidado de salud de las personas con base en sus recursos disponibles.

Es claro que una situación de pandemia genera un aumento de la demanda de la atención médica rápida y en corto plazo, generando una responsabilidad médica adicional que lleva a un incremento abrupto del consumo de insumos, una sobrecarga de trabajo del personal de salud, especialmente en el área de cuidados críticos, estudios de laboratorio, equipos de protección personal, y adecuación del sistema de salud para dar respuesta las nuevas necesidades. Esto deber realizarse direccionando los recursos, es decir posponiendo los procedimientos quirúrgicos programados, cerrando consultas no urgentes, paralizando los programas preventivos, derivando los recursos a las necesidades de la pandemia. Esta adecuación del sistema de salud, sumada al temor de los 
pacientes al contagio si salen del aislamiento genera el riesgo de dejar desplazadas o no atendidas las necesidades de los pacientes con enfermedades crónicas o agudas no relacionadas con la pandemia. Esto ha sido plasmado en el aumento de mortalidad en pacientes no-COVID (Appleby, 2020), o la dificultad en el retraso al acceso al sistema de salud (Masroor, 2020), aun en países desarrollados con altos niveles de vida, y sistemas de salud pública.

\section{Asignación de recursos}

La asignación de recursos consiste en la distribución de los limitados activos en sus diferentes usos según las necesidades de la sociedad. Existen distintas maneras de enfocar la distribución de recursos en forma justa y equitativa. La asignación de recursos se puede realizar en forma racional basada en análisis de parámetros costo-efectividad, costo-utilidad que consideran el beneficio terapéutico, por un lado, y los costos que implican el uso de esos recursos, por otro. En esta situación de pandemia la insuficiente evidencia actual sobre el beneficio terapéutico está basada en datos escasos, contradictorios y confusos, dificultando la toma de decisiones.

Existen distintos enfoques que sostienen la asignación justa y equitativa de los recursos dependiendo de cómo se consideren los parámetros, y el objetivo con el cual se aplican.

(Mooney, 1986) describe 6 parámetros de análisis que incluyen: 1) para cada uno lo mismo, considera como criterio de asignación la igualdad; 2) a cada uno según su mérito o logro, considera como criterio de asignación el esfuerzo que hace cada individuo en la sociedad; 3) a cada uno según su trabajo o contribución, considera como criterio de asignación los beneficios que se aportan a la sociedad; 4) a cada uno según su necesidad o requisitos, considera como criterio a la equidad; 5) a cada uno según su estado o posición, considera como criterio de asignación su posición en la sociedad; y 6) a cada uno según su contrato o acuerdos, considera como criterio de asignación los contratos previos que se han acordado con los diferentes sectores sociales. Estos parámetros pueden aplicarse en diferentes circunstancias, pero cuando se trata del derecho a la salud y del derecho a la vida es claro, es necesario que la base del análisis se centre en la equidad de acceso a los servicios de salud.

(Emanuel, y otros, 2020) por su parte propone cuatro valores a considerar en la asignación de recursos: 1) maximizar los beneficios producidos por los escasos recursos. Aquí la discusión se asienta en si el beneficio producido es el de salvar la mayoría de las vidas individuales (años de vida ganados-AVG), o salvar la mayoría de los años de vida al dar prioridad a los pacientes que probablemente sobrevivan más tiempo después del tratamiento (años de vida acumulados por calidad de vidaAVAC); 2) tratar a las personas por igual. En el caso de recursos escasos se plantea el conflicto si la asistencia se ofrece mediante una selección aleatoria, como como una lotería, o por una asignación por orden de llegada; 3) promover y recompensar el valor instrumental. Se orienta a favorecer a aquellos que más bienes pueden generar a la sociedad, ya sea que estos beneficios hayan sido producidos previa, durante o posteriormente a la pandemia; y 4) dar prioridad al peor de los casos. Si se considera como el peor de los casos a los más desfavorecidos, a las personas más jóvenes que no hayan pasado la mitad de sus vidas, o quienes tienen enfermedades más severas. Emanuel y cols. realizan un análisis que maximice los beneficios, de manera que los pacientes con un mejor pronóstico de años de vida acumulados por calidad de vida reciban más apoyo, ya que son quienes tendrían más beneficios que perder y quienes más puedan aportar a la sociedad.

Estos parámetros de análisis consecuencialista utilitaristas generan un dilema 
en el momento de decidir la asignación de recursos. Es decir, hay un deber de cuidar la salud y la vida del mayor número de personas en un sistema de salud con recursos limitados, pero también es necesario considerar variables consecuentes en la forma de asignar estos recursos a aquellos que tendrán una ventaja previa a recuperar su salud y mantener una buena calidad de vida posterior respecto de quienes ya tienen enfermedades previas que hagan considerar que su pronóstico de sobrevida o calidad de vida posterior no sea tan favorable ( Brown, Goodwin, Firth, \& Eyal, 2020). Por ejemplo, las recomendaciones de la University of Washington Medical Center define:

que la supervivencia general puede calificarse aún más como supervivencia saludable a largo plazo, reconociendo que esto representa una mayor ponderación de la supervivencia de pacientes jóvenes y en general sanos, que la de pacientes mayores con debilidad crónica. Dicha ponderación tiene un apoyo general en medicina y en la sociedad en general

Y contrapone esta visión con la del enfoque estándar de los médicos, que tienen como obligación ética defender y priorizar la atención de un paciente en particular. En esta perspectiva, el médico debería adoptar otros valores frente a su paciente en cuidado crítico que no constituyan su bienestar, sino el bienestar de la sociedad. En esta perspectiva si la pandemia se agrava, puede ser necesario adoptar un principio utilitario mediante el cual se deniegue el tratamiento hospitalario y las admisiones a algunos para que más personas puedan recibir la atención que necesitan (Challen, 2009). Entonces, si la lógica de la perspectiva utilitarista es promover el bien, ies realmente bueno prevalecer sólo el bien para la sociedad y no el bien individual, asignando solo los recursos a aquellos que tienen una ventaja para sobrevivir, y como consecuencia no sobrevivan aquellos con menos posibilidades 0 que se encuentren en situación de vulnerabilidad? Se vuelve cuestionable la utilidad cuando el beneficiario es un ser impersonal como la sociedad, sería un escenario en el que la equidad habría sido sacrificada en nombre de la eficiencia (Ne'eman A. 'I Will Not Apologize for My Needs' Even in a crisis, doctors should not abandon the principle of nondiscrimination, 2020). Esta perspectiva impersonalista del utilitarismo interpreta como valor moral fundamental la obligación de la sociedad hacia las futuras generaciones. No juzga la moralidad de estas decisiones en términos de su efecto sobre la persona en forma individual o específica, tampoco valora la vida humana en su naturaleza intrínseca, sino en la forma en que afecta a la sociedad (Heyd, 1992). Es necesario encontrar una alternativa que no pierda la necesidad de decidir cómo se realiza la asignación de los recursos escasos, pero que logre distribuirlos en forma justa y equitativa a todos los integrantes de la sociedad. EI profesional de la salud tiene la responsabilidad ética y legal de cuidar la salud de su paciente, aliviar su sufrimiento, y respetar sus derechos y deseos, en la medida de sus posibilidades. Los valores de la ética médica no pueden ser contrarios a los valores de la ética de la salud pública, ya que la salud pública tiene como deber promover la seguridad pública, proteger la salud de la comunidad, y asignar de manera equitativa los recursos limitados (Berlinger, y otros, 2020). Para lograr esta distribución equitativa de recursos es necesario que todas las personas, más allá de su capacidad de recuperar su salud, sean consideradas con igual valor.

\section{El personalismo y su visión antropológica}

Reich define la bioética como "Estudio sistemático de las dimensiones morales (incluyendo la visión moral, decisiones, conductas y políticas) de las ciencias de la vida y de la atención de la salud, empleando una variedad de metodologías éticas en un contexto interdisciplinar" (Reich, 1995). Con esta 
definición enmarca el objeto formalísimo con una visión ética pluralista, con una variedad de modelos éticos de referencia y una diversidad de teorías éticas que van a permitir la fundamentación del juicio ético desde distintas perspectivas. La variedad de modelos de análisis para justificar los juicios morales puede llevar a conclusiones aún contradictorias para analizar la licitud de los dilemas morales (DeGrazia, 1992).

El personalismo ontológicamente produce un quiebre en el análisis ético, ya que incorpora en su teoría de análisis del acto moral una definición antropológica del ser humano.

Tiene una visión del ser humano integral como ser trascendente, definiéndolo ontológicamente como una unidad sustancial, racional, libre, afectivo, espiritual, social, abierto a la trascendencia. En esta definición ontológica integra y no separa todos los aspectos del acto humano. Esta perspectiva integral del hombre lo diferencia de otras corrientes de análisis ético, en las que prepondera alguna característica del ser humano sobre otras (Beauchamp \& Childress, 2013). Es así, el aspecto racional del hombre permite el análisis con un enfoque consecuencialista-utilitarista, necesario para las decisiones de políticas de salud pública, siempre que el bienestar buscado se oriente a la protección de la vida y el bienestar de todas las personas, y no a un bienestar abstracto $y$ colectivo. El reconocimiento del hombre como ser libre, autodeterminado y autónomo, pero una libertad enmarcada en la responsabilidad de los actos. La valoración de los afectos, las relaciones personales, las circunstancias particulares que pueden condicionar, pero no determinar los actos morales. En resumen, esta visión integral del hombre no lo limita a alguna de sus características, sino que las integra en la búsqueda de la transcendencia del hombre más allá de sus circunstancias.

También define al ser humano axiológicamente, en el reconociendo de su dignidad inherente, dignidad que no es adquirida al completar su desarrollo físico, racional o emocional, ni otorgada por otros. Entiende que todos los seres humanos merecen respeto más allá de sus características físicas o ejercicio de sus cualidades humanas.

De esta manera, Sgreccia organiza esta teoría centrada en el principio de defensa de la vida física y sostiene este principio en el reconocimiento de la dignidad intrínseca, como valor ontológico objetivo, de la intangibilidad y sacralidad de la vida humana (Sgreccia, 2007).

Define en este principio al ser humano y en los tres principios siguientes analiza los actos humanos desde su teoría del acto moral, siendo la virtud la guía del juicio práctico. Entonces, en el principio de libertad-responsabilidad reconoce el ejercicio libre del acto humano, siendo la responsabilidad el marco moral que permitir vislumbrar en su accionar su finalidad real y los alcances, para sí y para otros. El principio totalidad-terapéutico, analiza la conducta humana $y$ sus consecuencias positivas $y$ negativas. Siendo el hombre una totalidad física, espiritual y moral, todas las decisiones que se tomen implican el discernimiento de los efectos positivos y negativos que la decisión contiene, y requiere la justificación de un balance positivo entre estos efectos. Finalmente, el principio de socialidad y subsidiariedad, que estudia en un doble camino el efecto del acto humano en la sociedad, y el efecto del comportamiento social en la persona humana. Este principio estipula que el ser humano es un ser social, que necesita en apoyo de toda la sociedad para su desarrollo en tres ejes: el eje individual, el cuidado de la propia vida; un horizontal, el cuidado de los otros; y un eje vertical, el apoyo de las instituciones superiores en el cuidado de la comunidad y de las personas con mayores necesidades. 


\section{Conclusiones}

- En un escenario de recursos escasos, incertidumbre en la toma de decisiones, riesgo del colapso del sistema de salud, evidencia dramática de la inequidad, surge la pregunta acerca del valor de la salud y qué estamos dispuestos a relegar o sacrificar para preservar este valor. La respuesta a esta pregunta no viene dada desde la infectología o la epidemiología o desde la política o economía de los países, es una respuesta de índole filosófica (Petrini \& Gainotti, 2008). Esta respuesta va a fundamentar la solución de los problemas que surgen al legislar las políticas de salud, de cuidado de la población general, y de administración justa y equitativa de los recursos en una situación intempestiva como una pandemia.

- La pandemia no tiene sólo un efecto en la salud de las personas o en la salud pública, las decisiones que se toman afectan a todas las instancias de la vida en sociedad. Por ello, requieren soluciones integrales, que incluyan transparencia, compromiso comunitario, integridad, y responsabilidad política en la toma de decisiones. El personalismo ontológico ofrece un marco de análisis que facilita el enfoque del cuidado de la sociedad en el cuidado de la persona, propone el desafío de trasladar el enfoque individualista y solitario a un enfoque comunitario. Enfoque comunitario que debe basarse en un único principio ordenador el reconocimiento y respeto por la dignidad de la persona.

\section{Bibliografía}

ACOG. (2003). The American College of Obstetricians and Gynecologists. Obtenido de Committee opinion 563 (Reaffirmed 2016): Ethical Issues in pandemic influenza planning concerning pregnant

women:

https://www.acog.org/clinical/clinical-

guidance/committee-

opinion/articles/2013/05/ethical-issues-in-

pandemic-influenza-planning-concerningpregnant-women

Appleby, J. (2020). What is happening to noncovid deaths? BMJ (Clinical research ed.). doi:https://doi.org/10.1136/bmj.m1607

Bauch, C. T., Lloyd-Smith, J. O., Coffee, M. P., \& Galvani, A. P. (2005). Dynamically modeling SARS and other newly emerging respiratory illnesses: past, present, and future.

Beauchamp, T., \& Childress , J. (2013). Principles of Biomedical Ethics. Oxford University Press, 7.

Berlinger, N., Wynia, M., Powell, T., Hester, D. M., Milliken, A., Fabi, R., . . . Piper Jenks, N. (2020). Ethical Framework for Health Care Institutions Responding to Novel Coronavirus SARS-CoV-2 (COVID-19) Guidelines for Institutional Ethics Services Responding to COVID-19. Managing Uncertainty, Safeguarding Communities, Guiding Practice. Obtenido de https://www.thehastingscenter.org/ethica Iframeworkcovid19/

Boeuf, P., Drummer, H. E., Richards, J. S., Scoullar, M. J., \& Beeson, J. G. (2016). The global threat of Zika virus to pregnancy: epidemiology, clinical perspectives, mechanisms, and impact. BMC medicine, 14(1).

doi:https://doi.org/10.1186/s12916-0160660-0

Brown, M. J., Goodwin, J., Firth, P., \& Eyal, N. (2020). Allocating Medical Resources in the Time of Covid-19. The New England journal of medicine. 
doi:https://doi.org/10.1056/NEJMc200966 6

Cascella, M., Rajnik, M., Cuomo, A., Dulebohn, S., \& Di Napoli, R. (2020). Features, Evaluation and Treatment Coronavirus (COVID-19). StatPearls. StatPearls Publishing.

Challen, K. (2009). Life or death decisions. Emerg Nurse.

China Medical Treatment Expert Group for Covid-19. (2020). Clinical Characteristics of Coronavirus Disease 2019 in China. The New England journal of medicine, 17081720.

doi:https://doi.org/10.1056/NEJMoa20020 32

Dashraath, P., Wong, J., Lim, M., Lim, L. M., Li, S., Biswas, A., . . . Su, L. L. (2020). Coronavirus disease 2019 (COVID-19) pandemic and pregnancy. American journal of obstetrics and gynecology, 22(6), 521-531. doi:https://doi.org/10.1016/j.ajog.2020.0 3.021

DeGrazia, D. (1992). Moving Forward in Bioethical Theory: Theories, Cases, and Specified Principlism. Journal of Medicine and Philosophy. doi:10.1093/jmp/17.5.511

Epidemiology (Cambridge, Mass.), 16(6), 791801.

doi:https://doi.org/10.1097/01.ede.00001 81633.80269.4c

Emanuel, E. J., Persad, G., Upshur, R., Thome, B., Parker, M., Glickman, A., ... Phillips, J. P. (2020). Fair Allocation of Scarce Medical Resources in the Time of Covid-19. he New England journal of medicine, 382(21),

2049-2055.
doi:https://doi.org/10.1056/NEJMsb20051 14

Hajra, A., Bandyopadhyay, D., \& Hajra, S. K. (2016). Zika Virus: A Global Threat to Humanity: A Comprehensive Review and Current Developments. North American journal of medical sciences, 8(3), 123128.

Hajra, A., Bandyopadhyay, D., Heise, L. R., Bhadra, R., Ball, S., \& Hajra, S. K. (2017). Zika and pregnancy: A comprehensive review. American journal of reproductive immunology (New York, N.Y. : 1989), $77(2)$.

doi:https://doi.org/10.1111/aji.12607

Heyd, D. (1992). Genethics Moral Issues in the Creation of People. The Regents of the University of California University of California Press Berkeley.

Homeland Security Council. (2005). Implementation Plan for the National Strategy for Pandemic Influenza. Obtenido de https://www.cdc.gov/flu/pandemicresources/pdf/pandemic-influenzastrategy-2005.pdf

Huang, C., Wang, Y., Li, X., Ren, L., Zhao, J., Hu, Y., . . . Cao, .. (2020). Clinical features of patients infected with 2019 novel coronavirus in Wuhan, China. Lancet 497-506.

doi:https://doi.org/10.1016/S0140-

6736(20)30183-5

Istituto Superiore di Sanit. (2020). Report sulle caratteristiche dei pazienti deceduti positivi a COVID-19 in Italia. Obtenido de https://www.epicentro.iss.it/coronavirus/b ollettino/Report-COVID201920 marzo.pdf 
Jones, D. S. (2020). History in a Crisis - Lessons for Covid-19. The New England journal of medicine, 382(18), 1681-1683. doi:https://doi.org/10.1056/NEJMp20043 61

Markel, H, H., Lipman, H. B., Navarro, J. A., Sloan, A., Michalsen, J. R., Stern, A. M., \& Cetron, M. S. (2007). Nonpharmaceutical interventions implemented by US cities during the 1918-1919 influenza pandemic. JAMA, 298(6), 644-654. doi:https://doi.org/10.1001/jama.298.6.64 4

Masroor, S. (2020). Collateral damage of COVID19 pandemic: Delayed medical care. Journal of cardiac surgery. doi:https://doi.org/10.1111/jocs.14638

Mooney, C. (1986). Criteria for Justice. American Journal of Economics and Sociology, $45(2), 223-233$.

Nathanson, N., \& Kew, O. M. (2010). From emergence to eradication: the epidemiology of poliomyelitis deconstructed. American journal of epidemiology, 1213-1229. doi:https://doi.org/10.1093/aje/kwq320

Ne'eman A. 'I Will Not Apologize for My Needs' Even in a crisis, doctors should not abandon the principle of nondiscrimination. (23 de Marzo de 2020). The New York Times. Obtenido de https://www.nytimes.com/2020/03/23/opi nion/coronavirus-ventilators-triagedisability.html

Organización Mundial de la Salud. (2020). Consideraciones relativas a la discapacidad durante el brote de COVID$19 . \quad$ Obtenido de https://www.who.int/docs/defaultsource/documents/disability/spanish- covid-19-disability-

briefing.pdf?sfvrsn $=30 d 726 b 1 \_2$

Pagliusi, S., Dennehy, M., Kim, H., \& DCVMN AGM Organizing Committee. (s.f.). Vaccines, inspiring innovation in health. Vaccine, 36(48), 7430-7437. doi:https://doi.org/10.1016/j.vaccine.2018 .05 .035

Petrini, C., \& Gainotti, S. (2008). A personalist approach to public-health ethics. Bulletin of the World Health.

Pringle, K. G., Kind, K. L., Sferruzzi-Perri, A. N., Thompson, J. G., \& Roberts, C. T. (2010). Beyond oxygen: complex regulation and activity of hypoxia inducible factors in pregnancy. Human reproduction update, 16(4), 415-431. doi: https://doi.org/10.1093/humupd/dmp046

Rasmussen, S. A., Jamieson, D. J., Macfarlane, K., Cragan, J. D., Williams, J., Henderson, Z., \& Grou, P. I. (2009). Pandemic influenza and pregnant women: summary of a meeting of experts. American journal of public health. doi:https://doi.org/10.2105/AJPH.2008.15 2900

Reich, W. T. (1995). Encyclopedia of Bioethics. Macmillan Publishing.

Schwartz, D. A., \& Graham, A. L. (2020). Potential Maternal and Infant Outcomes from (Wuhan) Coronavirus 2019-nCoV Infecting Pregnant Women: Lessons from SARS, MERS, and Other Human Coronavirus Infections. Viruses, 12(2). doi: https://doi.org/10.3390/v12020194

Sgreccia, E. (2007). Manual de Bioética: Fundamentos $y$ ética biomédica. Biblioteca de Autores Cristianos. 
World Health Organization . (2020). 2019 novel Coronavirus Global research and innovation forum: towards a research roadmap. Obtenido de https://www.who.int/blueprint/prioritydiseases/key-action/Roadmap-versionFINAL-for-WEB.pdf?ua =1
World Health Organization. (2020). Coronavirus disease 2019 (covid-19). Situation report 142.

Obtenido

de https://www.who.int/docs/defaultsource/coronaviruse/situationreports/20200610-covid-19-sitrep142.pdf?sfvrsn $=180898 \mathrm{~cd} \_2$ 